\title{
The Role of Shear Deformation in Laminated Plates with Curvilinear Fiber Paths and Embedded Defects
}

\author{
A. H. Akbarzadeh", M. Arian Nik ${ }^{*}$, D. Pasini ${ }^{\dagger}$ \\ Department of Mechanical Engineering, McGill University, Montreal, QC, H3A OC3, Canada
}

\begin{abstract}
Automated Fiber Placement (AFP) is an advanced technology used to manufacture laminated composites with curvilinear fiber paths. During the manufacturing, AFP generally leads to the formation of defects, e.g. gaps and overlaps, which impact the structural properties of a laminate. This paper focuses on the responses of moderately-thick laminate plates with embedded gaps and overlaps induced by AFP. We use higher-order shear deformation theories to study the role of shear deformation on the plate responses. A hybrid Fourier-Galerkin method is used to obtain a semi-analytic solution describing the structural behavior of the plate. The numeric results show that shear deformation has a more severe impact on the structural responses of a variable stiffness than on a constant stiffness plate. We find also that gaps deteriorate the structural performance, while overlaps improve it. Maps representing structural responses, in particular buckling vs. deflection and frequency vs. deflection, are generated to gain insight into the design of a variable stiffness laminate plate with defects.
\end{abstract}

Keywords: A. Structural composites, C. Multiscale modeling, C. Plate theory, C. Deformation, C. Buckling, Automated fiber placement

\section{Introduction}

Laminated composite structures have drawn special attention from a variety of sectors including aerospace, automotive, naval, and construction [1]. To achieve high strength-to-weight ratio,

\footnotetext{
* A.H. Akbarzadeh (hamid.akbarzadeh@mcgill.ca) and M. Arian Nik (mahdi.ariannik@mail.mcgill.ca) have contributed equally to this work.

† Corresponding author: damiano.pasini@mcgill.ca; Tel: (+1) 514-398-6295; Fax: (+1) 514-398-7365.
} 
high stiffness-to-weight ratio, fatigue strength, and resistance to corrosion, composite laminates are designed with either constant or variable stiffness. In a constant-stiffness design, a laminate has layers of straight fibers that have the highest stiffness and strength when loaded along the fiber direction. In a variable stiffness laminate, the fiber direction can be tailored to follow curvilinear fiber paths that best improve structural performance, such as buckling load [2-4], natural frequencies [5], and flexural stiffness [7]. Variable stiffness plates have also been demonstrated capable to offer trade-off properties that can concurrently optimize conflicting requirements, such as buckling load and in-plane stiffness [3, 8-10].

Several approaches exist in literature for the analysis of a laminated composite. Among them, equivalent single-layer (ESL) [11], three-dimensional (3D) elasticity [12], and multiple model methods [1] have been successfully used. In this paper, we use ESL theory to reduce the computational effort required for the analysis of a variable-stiffness composite. The simplest ESL theory is the classical laminated plate theory (CLPT), whereby the lines that before deformation are straight and normal to the mid-plane of the laminate are assumed to keep these characteristics even after deformation. An outcome of this hypothesis is the neglect of the deformation caused by transverse shear $\left(\sigma_{x z}, \sigma_{y z}\right)$ and transverse normal $\left(\sigma_{z z}\right)$ stresses. For moderately-thick composite laminates, first-order shear deformation theory (FSDT) and thirdorder shear deformation theory (TSDT) have been introduced to account for deformation caused by transverse shear [13]. FSDT assumes a constant shear strain through the thickness of a laminate and introduces a shear correction coefficient to compute transverse shear forces. TSDT, on the other hand, uses a quadratic function to describe transverse shear stresses through the thickness, with vanishing values at the top and bottom surfaces of the laminate [1]. FSDT and TSDT have been extensively used to model the structural mechanics of heterogeneous isotropic 
beams and plates [15-18]. For instance, ESL and 3D elasticity theories were applied to examine the buckling and post-buckling responses of laminated composites [20]. Another study focused on the resonance frequencies of free vibration of laminated composites with constant stiffness [22].

While there is a considerable amount of research that examines transverse shear deformation in constant stiffness composites, only a few studies look at variable stiffness laminates. One of these is that of Groh et al. [24], who studied via FSDT the impact of transverse shear deformation onto the flexural behavior of composite laminates with curvilinear fibers. It was shown that for a laminate with a length-to-thickness ratio of 10:1, an error of $43 \%$ may appear in the predicted transverse deflection if transverse shear stresses are neglected. Akhavan et al. $[5,7]$ used the finite element method and TSDT to study the natural frequency and large deflection of a variable stiffness laminate. It was found that a variable stiffness design can remarkably reduce the deflection of a plate compared to a constant stiffness design and might yield improvement in the natural frequency.

Only until recently, the impact of defects within variable stiffness laminates was overlooked in literature. In practice, however, the formation of defects, mainly gaps and/or overlaps, is unavoidable in variable stiffness laminate manufactured with Automated Fiber Placement (AFP). A method, namely "Defect layer", has been introduced $[8,10]$ to appraise the effect of gaps and overlaps on the critical buckling load and in-plane stiffness of a thin laminate. It has been shown that gaps and overlaps can significantly change the buckling load and in-plane stiffness. In a study by Li et al. [25], it is suggested that gap areas can be filled with fibers from the layers above the gap, and those fibers dip down into gap areas. While this may be true in certain instances, in this paper we assume that all gap areas are relatively small; as such, the fibers 
bridge over the gaps rather than dipping down. The outcome of this assumption is that 1) gap regions are less stiff than those filled with composite fibers, and 2) overlaps tend to generate stiffener-like features, which carry higher loads. With the recent use of AFP to build thick composite laminates for manned submersible applications [26], and moderately thick composite laminates in megawatt-scale wind turbine blades [27], this paper turns the attention to moderately-thick laminates with variable stiffness, with the aim of assessing the effect of gaps and overlaps on their structural performance. In particular, we use CLPT, FSDT, and TSDT theories to examine the impact of transverse shear deformation on the structural responses of a variable stiffness plate with embedded defects.

\section{Variable stiffness laminate}

A laminate plates manufactured with curvilinear fiber paths can be modelled by defining a reference fiber path along which the AFP machine places the first course. The subsequent fiber paths can be obtained by shifting the reference fiber path perpendicular to the steering direction. As a reference fiber path, we consider here one with constant curvature [28]. For this, the fiber orientation can be written as:

$$
\cos \theta=\cos T_{0}+\frac{|x|}{\rho}, R=\left|\frac{a / 2}{\left[\cos \left(T_{1}\right)-\cos \left(T_{0}\right)\right.}\right|
$$

where $\theta$ is the fiber orientation along the fiber path, $T_{0}$ and $T_{1}$ are respectively the fiber orientation at the plate midpoint and edges, $R$ is the turning radius along the path, and $a$ represents the plate width (Fig. 1a). A variable stiffness design is represented by $\left[<T_{0} \mid T_{1}>\right.$, where $T_{0}=T_{l}$ represents the case of straight fiber.

During the AFP process, the course width can be changed only by a discrete value, via either adding or dropping tows. As a result, defects in the form of gaps and overlaps emerge within the laminate. There are several strategies to add or drop a tow, such as complete gap and complete 
overlap strategies (Figures $1 \mathrm{~b}$ and 1c). [29]. To obtain the effective stiffness matrices of a variable stiffness laminate with embedded defects, we use here the defect layer method, recently introduced in $[8,10]$. According to this method, a defect layer is similar to a regular composite layer with modified material properties, or thickness proportional to the defect area percentage. Compared to a regular composite layer, a gap-modified defect layer has identical thickness and reduced elastic properties, whereas an overlap-modified defect layer is thicker than a regular composite layer and has its elastic properties. Interested readers may refer to [10] for more details about the defect-layer method.

\section{Problem definition and governing equations}

A rectangular laminated composite plate with length $a$, width $b$, and thickness $h$ is considered. We use here the CLPT to write displacement field as [1]:

$$
u(x, y, \mathrm{z}, t)=u_{0}(x, y, t)-z \frac{\partial w_{0}(x, y, t)}{\partial x}, v(x, y, \mathrm{z}, t)=v_{0}(x, y, t)-z \frac{\partial w_{0}(x, y, t)}{\partial y}, w(x, y, \mathrm{z}, t)=w_{0}(x, y, t)
$$

Where $(u, v, w)$ are the displacement components along $(x, y, z)$ coordinate axes and $\left(u_{0}, v_{0}, w_{0}\right)$ stands for the displacement components of the midplane $(z=0)$. Using the TSDT, the displacement field for a laminated plate can be written as $[1,13]$ :

$$
\begin{gathered}
u(x, y, z, t)=u_{0}(x, y, t)+z \phi_{x}(x, y, t)-c_{1} z^{3}\left(\phi_{x}(x, y, t)+\frac{\partial w_{0}(x, y, t)}{\partial x}\right) \\
v(x, y, z, t)=v_{0}(x, y, t)+z \phi_{y}(x, y, t)-c_{1} z^{3}\left(\phi_{y}(x, y, t)+\frac{\partial w_{0}(x, y, t)}{\partial y}\right), w(x, y, z, t)=w_{0}(x, y, t)
\end{gathered}
$$

where $\phi_{x}$ and $\phi_{y}$ represent rotations about the $y$ and $x$ axes, respectively, and $c_{1}=4 / 3 h^{2}$. It is worth mentioning that by setting $c_{1}=0$ Eq. (3) reduces to the FSDT displacement field formulation. For small strains and moderate rotations, the von-Karman strains in terms of the FSDT/TSDT displacement field given in Eq. (3) are written as follows [13]: 


$$
\begin{gathered}
\left\{\begin{array}{c}
\varepsilon_{x x} \\
\varepsilon_{y y} \\
\gamma_{x y}
\end{array}\right\}=\left\{\begin{array}{c}
u_{0, x}+\frac{1}{2} w_{0, x}{ }^{2} \\
v_{0, y}+\frac{1}{2} w_{0, y}{ }^{2} \\
u_{0, y}+v_{0, x}+w_{0, x} w_{0, y}
\end{array}\right\}+z\left\{\begin{array}{c}
\phi_{x, x} \\
\phi_{y, y} \\
\phi_{x, y}+\phi_{y, x}
\end{array}\right\}-c_{1} z^{3}\left\{\begin{array}{c}
\phi_{x, x}+w_{0, x x} \\
\phi_{y, y}+w_{0, y y} \\
\phi_{x, y}+\phi_{y, x}+2 w_{0, x y}
\end{array}\right\} \\
\left\{\begin{array}{l}
\gamma_{y z} \\
\gamma_{x z}
\end{array}\right\}=\left\{\begin{array}{l}
\phi_{y}+w_{0, y} \\
\phi_{x}+w_{0, x}
\end{array}\right\}-c_{2} z^{2}\left\{\begin{array}{l}
\phi_{y}+w_{0, y} \\
\phi_{x}+w_{0, x}
\end{array}\right\}
\end{gathered}
$$

where the comma represents the partial differentiation operator, and $c_{2}=3 c_{1}$. Substituting $\phi_{x}=-w_{0, x}$ and $\phi_{y}=-w_{0, y}$ into Eq. (4) eliminates transverse shear strains and leads to the CLPT formulation. The equations of motion using the CLPT, obtained by the principle of virtual displacement, are written as [1]:

$$
\begin{gathered}
N_{x x, x}+N_{x y, y}=I_{0} \ddot{u}_{0}-I_{1} \ddot{w}_{0, x}, \quad N_{x y, x}+N_{y y, y}=I_{0} \ddot{v}_{0}-I_{1} \ddot{w}_{0, y} \\
M_{x x, x x}+2 M_{x y, x y}+M_{y y, y y}+\hat{N}\left(w_{0}\right)+q(x, y)=I_{0} \ddot{w}_{0}-I_{2}\left(\ddot{w}_{0, x x}+\ddot{w}_{0, y y}\right)+I_{1}\left(\ddot{u}_{0, x}+\ddot{v}_{0, y}\right)
\end{gathered}
$$

while the FSDT/TSDT equations of motion are expressed as [13]:

$$
\begin{gathered}
N_{x x, x}+N_{x y, y}=I_{0} \ddot{u}_{0}+J_{1} \ddot{\phi}_{x}-c_{1} I_{3} \ddot{w}_{0, x}, N_{x y, x}+N_{y y, y}=I_{0} \ddot{v}_{0}+J_{1} \ddot{\phi}_{y}-c_{1} I_{3} \ddot{w}_{0, y} \\
\bar{Q}_{x, x}+\bar{Q}_{y, y}+c_{1}\left(P_{x x, x x}+2 P_{x y, x y}+P_{y y, y y}\right)+\hat{N}\left(w_{0}\right)+q(x, y)=I_{0} \ddot{w}_{0}-c_{1}^{2} I_{6}\left(\ddot{w}_{0, x x}+\ddot{w}_{0, y y}\right) \\
+c_{1}\left(I_{3}\left(\ddot{u}_{0, x}+\ddot{v}_{0, y}\right)+J_{4}\left(\ddot{\phi}_{x, x}+\ddot{\phi}_{y, y}\right)\right) \\
\bar{M}_{x x, x}+\bar{M}_{x y, y}-\bar{Q}_{x}=J_{1} \ddot{u}_{0}+K_{2} \ddot{\phi}_{x}-c_{1} J_{4} \ddot{w}_{0, x}, \bar{M}_{x y, x}+\bar{M}_{y y, y}-\bar{Q}_{y}=J_{1} \ddot{v}_{0}+K_{2} \ddot{\phi}_{y}-c_{1} J_{4} \ddot{w}_{0, y}
\end{gathered}
$$

Where

$$
\begin{gathered}
\left(N_{\alpha \beta}, M_{\alpha \beta}, P_{\alpha \beta}\right)=\int_{-\frac{h}{2}}^{\frac{h}{2}} \sigma_{\alpha \beta}\left(1, z, z^{3}\right) d z,\left(Q_{\alpha}, R_{\alpha}\right)=c_{f} \int_{-\frac{h}{2}}^{\frac{h}{2}} \sigma_{\alpha \beta}\left(1, z^{2}\right) d z \\
\bar{M}_{\alpha \beta}=M_{\alpha \beta}-c_{1} P_{\alpha \beta}, \bar{Q}_{\alpha}=Q_{\alpha}-c_{2} R_{\alpha}, I_{i}=\int_{-\frac{h}{2}}^{\frac{h}{2}} \rho z^{i} d z
\end{gathered}
$$




$$
J_{i}=I_{i}-c_{1} I_{i+2}, K_{2}=I_{2}-2 c_{1} I_{4}+c_{1}^{2} I_{6}(i=0,1, \ldots, 6),\left\{\begin{array}{c}
c_{2} \neq 0, c_{f}=1 \text { TSDT } \\
c_{2}=0 \text { FSDT }
\end{array}\right.
$$

where $q(x, y)$ is the distributed transverse load at the bottom/top surface of the plate and $\alpha$ and $\beta$ take the symbols $x$ and $y, \sigma_{\alpha \beta}$ is the second Piola-Kirchhoff stress components, $c_{f}$ is the shear correction factor, $\rho$ is the mass density, and the superposed dot on a variable denotes the time derivation. It is worth noting that the determination of the shear correction factor $\left(c_{f}\right)$ for FSDT is cumbersome since it depends on lamination, geometric parameters, loading, and boundary conditions. We consider three values, commonly used in literature for FSDT analysis, $c_{f}=1, c_{f}=5 / 6$, and $c_{f}=3 / 4$. To conduct a bifurcation buckling analysis, the nonlinear in-plane force resultant $\hat{N}\left(w_{0}\right)$ should be written as $[1,13]$ :

$$
\hat{N}\left(w_{0}\right)=\left(N_{x x} w_{0, x}+N_{x y} w_{0, y}\right)_{, x}+\left(N_{x y} w_{0, x}+N_{y y} w_{0, y}\right)_{, y}
$$

The stiffness matrices, required for the structural analysis, are also defined as:

$$
\left(A_{i j}, B_{i j}, D_{i j}, E_{i j}, F_{i j}, H_{i j}\right)=\int_{-\frac{h}{2}}^{\frac{h}{2}} \bar{Q}_{i j}\left(1, z, z^{2}, z^{3}, z^{4}, z^{6}\right) d z
$$

where $\bar{Q}_{i j}$ is the transformed plane stress-reduced stiffness. The governing equations of CLPT and TSDT, which are not given here for the sake of brevity, are derived by using Eqs. (2) through (9). Herein, the nonlinear terms for static as well as free and forced vibration analyses are omitted; only the nonlinear in-plane force resultant $\hat{N}\left(w_{0}\right)$ is retained for buckling analysis. We note that in contrast to constant stiffness composites, here the elements $A_{i j}, B_{i j}, D_{i j}, E_{i j}, F_{i j}$, and $H_{i j}$ of the stiffness matrices are a function of the spatial coordinates $(x, y)$. As a result, the governing equations of motion for variable stiffness laminates are dissimilar from the conventional governing equations of a constant stiffness composite. 


\section{Methodology}

In this paper, we consider simply-supported boundary conditions (SS-1) [1]. Due to the presence of the derivatives of the stiffness matrices in the governing differential equations, the Galerkin method along with the Fourier series expansion are used to develop a semi-analytic solution. For displacement fields in FSDT/TSDT analysis, we consider the Fourier series expansions that satisfy the SS-1 simply-supported boundary conditions $[1,16]$ :

$$
\left\{\begin{array}{l}
u_{0}(x, y, t) \\
v_{0}(x, y, t) \\
w_{0}(x, y, t) \\
\phi_{x}(x, y, t) \\
\phi_{y}(x, y, t)
\end{array}\right\}=\sum_{n=1}^{n_{y}} \sum_{m=1}^{m_{x}}\left\{\begin{array}{l}
U_{m n}(t) \cos \left(r_{m} x\right) \sin \left(r_{n} y\right) \\
V_{m n}(t) \sin \left(r_{m} x\right) \cos \left(r_{n} y\right) \\
W_{m n}(t) \sin \left(r_{m} x\right) \sin \left(r_{n} y\right) \\
X_{m n}(t) \cos \left(r_{m} x\right) \sin \left(r_{n} y\right) \\
Y_{m n}(t) \sin \left(r_{m} x\right) \cos \left(r_{n} y\right)
\end{array}\right\}
$$

where $r_{m}=m \pi / a, r_{n}=n \pi / b, U_{m n}, V_{m n}, W_{m n}, X_{m n}$, and $Y_{m n}$ are unknown coefficients that should be determined to satisfy the governing differential equations, being $n_{y}$ and $m_{x}$ arbitrary integers of the summation. Using the Galerkin method and the approximate displacement field in Eq. (10), we can solve the governing differential equations for the FSDT/TSDT analysis [1, 16]:

$$
\int_{y=0}^{b} \int_{x=0}^{a}\left\{\begin{array}{l}
R_{1}\left(U_{m n}, V_{n n}, W_{m n}, X_{m n}, Y_{m n}\right) \cos \left(r_{p} x\right) \sin \left(r_{q} y\right) \\
R_{2}\left(U_{m n}, V_{m n}, W_{m n}, X_{m n}, Y_{m n}\right) \sin \left(r_{p} x\right) \cos \left(r_{q} y\right. \\
R_{3}\left(U_{m n}, V_{m n}, W_{m n}, X_{m n}, Y_{m n}\right) \sin \left(r_{p} x\right) \cos \left(r_{q} y\right. \\
R_{4}\left(U_{m n}, V_{m n}, W_{m n}, X_{m n}, Y_{m n}\right) \sin \left(r_{p} x\right) \cos \left(r_{q} y\right. \\
R_{5}\left(U_{m n}, V_{n n}, W_{m n}, X_{m n}, Y_{m n}\right) \sin \left(r_{p} x\right) \cos \left(r_{q} y\right.
\end{array}\right\} d x d y=0 \quad\left(p=1,2, \ldots, m_{x}, q=1,2, \ldots, n_{y}\right)
$$

where $R_{i}(i=1, \ldots, 5)$ are the residuals of the governing differential equations for the admissible displacement field (Eq. (10)). Substituting Eq. (10) into governing equations and then applying the Galerkin formulation (Eq. (11)), leads to an expanded formulation, which results in a $\left(5 \times m_{x} \times n_{y}\right) \times\left(5 \times m_{x} \times n_{y}\right)$ system of differential equations:

$$
\left[K_{T S D T}\right]\left\{\Delta_{T S D T}\right\}+\left[M_{T S D T}\right]\left\{\ddot{i}_{T S D T}\right\}=\left\{F_{T S D T}\right\}
$$


where $\left\{\Delta_{T S D T}\right\}^{T}=\left\{\begin{array}{lllllllllll}U_{11} & V_{11} & W_{11} & X_{11} & Y_{m n} & \ldots & U_{m_{x} n_{y}} & V_{m_{x} n_{y}} & W_{m_{x} n_{y}} & X_{m_{x} n_{y}} & Y_{m_{x} n_{y}}\end{array}\right\}, K_{T S D T}$ and $M_{T S D T}$ are stiffness and mass matrices, and $F_{T S D T}$ represents the mechanical force vector. If no $\hat{N}\left(w_{0}\right)$ exists, the system of governing differential equations can be solved for static and free and forced vibration analyses. For the transient analysis, the Newmark integration procedure can be adopted $[1,13]$. If $\hat{N}\left(w_{0}\right)$ is present, however, a bifurcation analysis is necessary to obtain the critical buckling load [1]. For the CLPT analysis, applying the abovementioned Fourier-Galerkin methodology results in a $\left(3 \times m_{x} \times n_{y}\right) \times\left(3 \times m_{x} \times n_{y}\right)$ system of differential equations. The derived differential equations are solved via MATLAB scripts developed for variable stiffness composite laminates with embedded defects.

\section{Results and discussion}

To validate the methodology presented in Section 4, we first compare the results available in literature for static bending, buckling, and natural vibration of plates with those obtained for constant stiffness laminated composites. Then, we examine the impact of shear stresses and manufacturing defects on structural responses of variable stiffness plates.

\subsection{Result validation}

We consider a simply-supported plate with constant stiffness, symmetric cross-ply $[0 / 90]_{s}$ and planar square $(a=b=1 m)$ geometry. Its material properties are $E_{1}=175 G P a, E_{2}=7 G P a$, $G_{12}=G_{13}=3.5 G P a, G_{23}=1.4 G P a$, and $v_{12}=0.25$. The second row of Table 1 shows the results of the dimensionless midpoint deflection $\left(\bar{\omega}=\frac{\omega a^{2}}{h} \sqrt{\frac{\rho}{E_{2}}}\right)$ of the plate subjected to a distributed transverse load $\left(q=q_{0} \sin \left(r_{m} x\right) \sin \left(r_{n} y\right)\right)$. The results show that all the predictions are very close to those provided in [1]. For a plate with the length-to-thickness ratio of $a / h=4$, CLPT 
underpredicts the maximum deflection by $74 \%$ compared to the solution obtained with $3 \mathrm{D}$ elasticity, whereas the FSDT and TSDT show a discrepancy of $12.5 \%$ and 3\%, respectively. The third and fourth rows of Table 1 show the dimensionless uniaxial critical buckling load $\left(\bar{N}=\frac{N_{c r} a^{2}}{E_{2} h^{3}}\right)$ and fundamental frequency $\left(\bar{\omega}=\frac{\omega a^{2}}{h} \sqrt{\frac{\rho}{E_{2}}}\right)$ as a function of the modulus ratio $E_{1} / E_{2}$ for $a / h=5$ and 10, where $G_{12}=G_{13}=0.6 E_{2}, G_{23}=0.5 E_{2}$, and $v_{12}=0.25$. Similar to the case of maximum deflection, the application of FSDT/TSDT leads to more accurate results for critical buckling load and fundamental frequency. The results given in Table 1 indicate that the difference between the results predicted by FSDT and TSDT is much more evident in the maximum deflection than in the critical buckling load and fundamental frequency.

\subsection{Structural responses of variable stiffness laminates}

In this section, we use CLPT, FSDT, and TSDT to study the influence of manufacturing defects on the structural responses of variable stiffness plates. We consider a square plate $(a=b=1 \mathrm{~m})$ made of 16-ply balanced and symmetric laminate with a variable stiffness design of $[ \pm<58 \mid 39>]_{4 s}$. The material properties of prepreg composites are: $E_{1}=143 \mathrm{GPa}, E_{2}=9.1 \mathrm{GPa}, G_{12}=4.82 \mathrm{GPa}$, $G_{13}=G_{23}=4.9 \mathrm{GPa}, \quad v_{12}=0.3, \rho=1500 \mathrm{Kg} / \mathrm{m}^{3}$ while the material properties of the resin are specified as: $E_{1}=3.72 \mathrm{GPa}, E_{2}=3.72 \mathrm{GPa}, G_{12}=G_{13}=G_{23}=1.43 \mathrm{GPa}, v_{12}=0.3, \rho=1100 \mathrm{Kg} / \mathrm{m}^{3}$. The structural responses of the plate, including static deflection, uniaxial critical buckling load, and fundamental frequency are compared in Table 2 with those of a quasi-isotropic (QI) plate, here selected as a baseline. The plate is subjected to a uniform transverse loading, $q(x, y)=q_{0}$, for static analysis. 


\subsubsection{Static analysis}

As shown in Table 2, all ESL theories predict very close maximum deflection for a very thin plate $(a / h=200)$. However for the length-to-thickness ratio $a / h=20$, discrepancies up to $7 \%$ emerge from CLPT and TSDT results for the maximum out-of-plane deflection. For a very thick laminate with $a / h=5$, differences are greater than $55 \%$. Furthermore, the discrepancy between ESL theories depends on the embedded manufacturing defects. For instance, for a plate with $a / h=10$, incorporating the effect of overlap increases the deviation by about $3 \%$ compared to the defect-free case, whereas considering the effect of gap decreases the discrepancy by $2 \%$. These trends can be attributed to the emergence of overlaps, which are thickness build-ups that decrease the overall length-to-thickness ratio of the plate in comparison with the defect-free case. On the other hand, a variable stiffness plate with gaps has effective elastic properties lower than a defect-free plate with higher length-to-thickness ratio.

\subsubsection{Buckling analysis}

Table 2 also shows significant discrepancies between the critical buckling load predicted with CLPT and TSDT for increasingly thicker plates. In particular for a plate with $a / h=20$ and $a / h=5$, the discrepancies between results obtained with CLPT and TSDT increase from $7 \%$ to $177 \%$ respectively. Furthermore, Table 2 reveals that shear stresses have an impact on the buckling load severer than that on the maximum out-of-plane deflection. For example for a plate with $a / h=10$, the difference between the CLPT and TSDT results for the maximum deflection is $23 \%$, while this value is greater than $33 \%$ for buckling. We can also observe that - compared to a defect-free case - gaps embedded in a variable stiffness plate reduce the buckling load, whereas overlaps increase it. For $a / h=10$, the buckling load reduces by $8 \%$ for a plate with gaps, whereas it increases by $31 \%$ for a plate with overlaps. Concerning the effect of the shear correction factor 
in FSDT, the results in Table 2 show that $c_{f}=5 / 6$ leads to the most accurate results compared to the other correction factors.

\subsubsection{Free vibration analysis}

From a comparison of CLPT and TSDT results for fundamental frequency (Table 2), a discrepancy greater than $4 \%$ can be observed for a plate with $a / h=20$; this value increases up to $48 \%$ for a thick plate with $a / h=5$. The differences between CLPT and TSDT is the least for fundamental frequency, followed by maximum out-of-plane deflection and critical buckling load. Table 2 shows also that variable stiffness plates with gaps have a fundamental frequency lower than defect-free laminates, whereas plates with overlaps have a higher value. The presence of gaps in a variable stiffness plate with $a / h=10$, for example, reduces the fundamental frequency by $3 \%$, while overlaps increase it by $10 \%$ when compared to a defect-free case.

\subsection{Influence of curvilinear fiber path}

This section examines the impact of $T_{0}$ and $T_{1}$ on the structural responses of a variable stiffness plate. For the plate here under investigation, Figure 2 illustrates the area percentages for gaps and overlaps as a function of $T_{0}$ and $T_{1}$ over the entire design space. The white areas represent plate designs that do not satisfy the manufacturing constraint, i.e., the minimum turning radius of $0.635 \mathrm{~m}$ imposed by a typical AFP machine. For straight-fiber laminates $\left(T_{0}=T_{1}\right)$, no gaps or overlaps appear in the laminate, whereas the area percentage for both gaps and overlaps increases if the difference between $T_{0}$ and $T_{1}$ becomes larger.

We examine a plate with $a / h=10$ for different manufacturing strategies. Figure 3 illustrates its critical buckling load and fundamental frequency versus maximum deflection, each normalized by the corresponding values of a quasi-isotropic laminate. As opposed to a constant stiffness 
design, whose response domain is represented by a line, a variable stiffness design yields to domains of a larger extent. Furthermore in comparison with the results obtained for a defect-free plate, overlaps shift the response domain towards a higher buckling load and lower maximum deflection. On the other hand, gaps shift the domain towards a lower buckling load and higher deflection. This behaviour is attributed to the effect of overlaps, which are thickness build-ups that tend to stiffen the plate along the fiber path, thereby improving its structural responses. In contrast gaps, resin-rich areas with reduced mechanical properties, deteriorate the structural responses of the plate. As shown in Fig. 2a, a complete gap strategy yields a large amount of gaps. In contrast, a complete overlap strategy results in an amount of overlap that continuously increases as the fiber path deviates from the straight path. As a result, a remarkable difference exists between the boundary of the feasible domain of a plate with gaps and the one with straight fibers. A similar behaviour can be observed in the domain fundamental frequency versus maximum deflection. From a comparison of Figs. 3a and 3b, we observe that the bucklingdeflection domain for a defect-free plate is significantly larger than the corresponding frequencydeflection domain.

\section{Concluding Remarks}

This paper has examined the effect of transverse shear deformation and embedded manufacturing defects on the structural responses of a variable stiffness plate made by AFP. Static bending, buckling, and free vibration have been studied. For moderately thick plates with length-tothickness ratio $a / h=10$, major differences emerge between CLPT and TSDT predictions. In particular, discrepancies up to $23 \%, 33 \%$, and $15 \%$ are observed for the maximum out-of-plane deflection, critical buckling load, and fundamental frequency. The results obtained in this paper show the important role played by shear deformation in thick plates with curvilinear fibers. We 
have also highlighted that the discrepancy between ESL theories depends on the amount of the embedded defects. In a static bending analysis of a plate with $a / h=10$ where the effects of overlaps are considered in the analysis, the maximum deflection calculated with CLPT diverges up to $26 \%$ from that predicted by TSDT. On the other hand, accounting for the effect of gaps results in about $22 \%$ difference. Finally maps have been presented to show how the structural responses of a defected plate changes with respect to a defect-free plate.

\section{References}

[1] Reddy JN. Mechanics of laminated composite plates and shells: theory and analysis: CRC press, 2003.

[2] Alhajahmad A, Abdalla MM, Gürdal Z. Optimal design of tow-placed fuselage panels for maximum strength with buckling considerations. J. Aircraft. 2010;47:775-82.

[3] Arian Nik M, Fayazbakhsh K, Pasini D, Lessard L. Surrogate-based multi-objective optimization of a composite laminate with curvilinear fibers. Compos. Struct. 2012;94:2306-13.

[4] Gürdal Z, Tatting BF, Wu C. Variable stiffness composite panels: effects of stiffness variation on the in-plane and buckling response. Compos. Part A: Appl. Sci. Manufactur. 2008;39:911-22.

[5] Akhavan H, Ribeiro P. Natural modes of vibration of variable stiffness composite laminates with curvilinear fibers. Compos. Struct. 2011;93:3040-7.

[7] Akhavan H, Ribeiro P, de Moura M. Large deflection and stresses in variable stiffness composite laminates with curvilinear fibres. Int. J. Mech. Sci. 2013;73:14-26.

[8] Arian Nik M, Fayazbakhsh K, Pasini D, Lessard L. Optimization of variable stiffness composites with embedded defects induced by Automated Fiber Placement. Compos. Struct. 2014;107:160-6. 
[9] Arian Nik M, Fayazbakhsh K, Pasini D, Lessard L. A comparative study of metamodeling methods for the design optimization of variable stiffness composites. Compos. Struct. 2014; 107:494-501.

[10] Fayazbakhsh K, Arian Nik M, Pasini D, Lessard L. Defect layer method to capture effect of gaps and overlaps in variable stiffness laminates made by automated fiber placement. Compos. Struct. 2013;97:245-51.

[11] Komeili A, Akbarzadeh AH, Doroushi A, Eslami MR. Static analysis of functionally graded piezoelectric beams under thermo-electro-mechanical loads. Adv. Mech. Eng. 2011;2011.

[12] Zhong Z, Yu T. Analytical solution of a cantilever functionally graded beam. Compos. Sci. Tech. 2007;67:481-8.

[13] Reddy J. Analysis of functionally graded plates. Int. J. Num. Meth. Eng. 2000;47:663-84.

[15] Akbarzadeh A, Abbasi M, Eslami M. Dynamic analysis of functionally graded plates using the hybrid Fourier-Laplace transform under thermomechanical loading. Meccanica. 2011;46:1373-92.

[16] Akbarzadeh A, Abbasi M, Eslami M. Coupled thermoelasticity of functionally graded plates based on the third-order shear deformation theory. Thin-Walled Struct. 2012;53:141-55.

[17] Akbarzadeh A, Zad SH, Eslami M, Sadighi M. Mechanical behaviour of functionally graded plates under static and dynamic loading. Proc. IMechE, Part C: J. Mech. Eng. Sci. 2011;225:32633.

[18] Kiani Y, Akbarzadeh A, Chen Z, Eslami M. Static and dynamic analysis of an FGM doubly curved panel resting on the Pasternak-type elastic foundation. Compos. Struct. 2012;94:2474-84. 
[20] Han S-C, Lee S-Y, Rus G. Postbuckling analysis of laminated composite plates subjected to the combination of in-plane shear, compression and lateral loading. Int. J. Solids Struct. 2006;43:5713-35.

[22] Aydogdu M, Timarci T. Vibration analysis of cross-ply laminated square plates with general boundary conditions. Compos. Sci. Tech. 2003;63:1061-70.

[24] Groh R, Weaver P, White S, Raju G, Wu Z. A 2D equivalent single-layer formulation for the effect of transverse shear on laminated plates with curvilinear fibres. Compos. Struct. 2013;100:464-78.

[25] Li X, Hallet SR, Wisnom MR. Modelling the effect of gaps and overlaps in automated fibre placement (AFP) manufactured laminates. 19th Int. Conf. Compos. Mater. 2013.

[26] New manned submersible to feature carbon fiber composite hull. Composites World 2013.

[27] Griffin DA, Ashwill TD. Alternative composite materials for megawatt-scale wind turbine blades: design considerations and recommended testing. ASME 2003 Wind Energy Symp.: 2003. p. 191-201.

[28] Blom AW, Lopes CS, Kromwijk PJ, Gurdal Z, Camanho PP. A theoretical model to study the influence of tow-drop areas on the stiffness and strength of variable-stiffness laminates. J. Comp. Mater. 2009;43:403-25.

[29] Tatting BF, Gürdal Z. Automated finite element analysis of elastically-tailored plates. NASA contractor report no NASA/CR-2003-212679. 2003.

Table 1: Dimensionless maximum deflection, critical buckling load, and fundamental frequency of a simplysupported, symmetric, and cross-ply $[0 / 90]_{s}$ plate. 


\begin{tabular}{|c|c|c|c|c|c|c|c|c|c|}
\hline & $a / h$ & $E_{1} / E_{2}$ & TSDT & $\mathbf{T S D T}^{[1]}$ & FSDT $^{*}$ & FSDT $^{*[1]}$ & CLPT & $\mathbf{C L P T}^{[1]}$ & $\begin{array}{c}\text { 3D } \\
\text { Elasticity }^{[1]}\end{array}$ \\
\hline \multirow[t]{2}{*}{$\bar{w} \times 100$} & 4 & \multirow[t]{2}{*}{25} & 1.8937 & 1.894 & 1.7091 & 1.71 & 0.4311 & 0.4311 & 1.954 \\
\hline & 10 & & 0.7146 & 0.715 & 0.6625 & 0.6625 & 0.4311 & 0.4311 & 0.743 \\
\hline \multirow[t]{2}{*}{$\bar{N}$} & \multirow[t]{2}{*}{10} & 20 & 15.2984 & 15.298 & 15.3513 & 15.351 & 19.7124 & 19.712 & 15.019 \\
\hline & & 40 & 23.34 & 23.34 & 23.4529 & 23.453 & 36.1597 & 36.16 & 22.881 \\
\hline \multirow[t]{2}{*}{$\bar{\omega}$} & \multirow[t]{2}{*}{5} & 20 & 9.5625 & 9.526 & 9.566 & 9.567 & 13.5108 & 13.511 & 9.56 \\
\hline & & 40 & 10.821 & 10.787 & 10.8529 & 10.854 & 18.2989 & 18.299 & 10.752 \\
\hline
\end{tabular}

\footnotetext{
${ }^{*}$ The correction factor is $c_{f}=5 / 6$.
} 
Table 2: Dimensionless maximum deflection $(\bar{w} \times 100)$ under uniform static load, dimensionless critical uniaxial buckling load $\bar{N}$, and dimensionless fundamental frequency $\bar{\omega}$. (QI: Quasi Isotropic)

\begin{tabular}{|c|c|c|c|c|c|c|c|c|}
\hline \multirow{2}{*}{$\begin{array}{l}\text { Structural } \\
\text { responses }\end{array}$} & \multirow{2}{*}{$a / h$} & \multirow{2}{*}{ Layup } & \multirow{2}{*}{$\begin{array}{l}\text { Manufacturin } \\
\text { g defects }\end{array}$} & \multirow{2}{*}{ CLPT } & \multicolumn{3}{|c|}{ FSDT } & \multirow{2}{*}{ TSDT } \\
\hline & & & & & $c_{f}=1$ & $c_{f}=5 / 6$ & $c_{f}=3 / 4$ & \\
\hline \multirow{16}{*}{$\bar{w} \times 100$} & \multirow{4}{*}{5} & QI & - & 0.6970 & 1.2974 & 1.4174 & 1.4973 & 1.3968 \\
\hline & & \multirow{3}{*}[\pm<58|39>]{$_{4 \mathrm{~s}}$} & Defect-free & 0.5787 & 1.1784 & 1.2989 & 1.3793 & 1.2902 \\
\hline & & & Complete gap & 0.6483 & 1.2474 & 1.3679 & 1.4482 & 1.3601 \\
\hline & & & Complete & 0.4233 & 0.9651 & 1.0738 & 1.1464 & 1.0139 \\
\hline & \multirow{4}{*}{10} & QI & - & 0.6970 & 0.8473 & 0.8773 & 0.8973 & 0.8735 \\
\hline & & \multirow{3}{*}[\pm<58|39>]{$_{4 \mathrm{~s}}$} & Defect-free & 0.5787 & 0.7269 & 0.7569 & 0.7769 & 0.7567 \\
\hline & & & Complete gap & 0.6483 & 0.7963 & 0.8263 & 0.8463 & 0.8262 \\
\hline & & & Complete & 0.4233 & 0.5578 & 0.5848 & 0.6029 & 0.5739 \\
\hline & \multirow{4}{*}{20} & QI & - & 0.6970 & 0.7346 & 0.7421 & 0.7471 & 0.7413 \\
\hline & & \multirow{3}{*}[\pm<58|39>]{$_{4 \mathrm{~s}}$} & Defect-free & 0.5787 & 0.6145 & 0.6219 & 0.6269 & 0.6223 \\
\hline & & & Complete gap & 0.6483 & 0.6841 & 0.6915 & 0.6965 & 0.6918 \\
\hline & & & Complete & 0.4233 & 0.4565 & 0.4632 & 0.4677 & 0.4606 \\
\hline & \multirow{4}{*}{200} & QI & - & 0.6970 & 0.6974 & 0.6975 & 0.6975 & 0.6975 \\
\hline & & \multirow{3}{*}[\pm<58|39>]{$_{4 \mathrm{~s}}$} & Defect-free & 0.5787 & 0.5775 & 0.5775 & 0.5776 & 0.5779 \\
\hline & & & Complete gap & 0.6483 & 0.6471 & 0.6472 & 0.6472 & 0.6475 \\
\hline & & & Complete & 0.4233 & 0.4232 & 0.4232 & 0.4233 & 0.4230 \\
\hline \multirow{16}{*}{$\bar{N}$} & \multirow{4}{*}{5} & QI & - & 22.944 & 11.014 & 9.6400 & 8.8997 & 10.020 \\
\hline & & \multirow{3}{*}[\pm<58|39>]{$_{4 \mathrm{~s}}$} & Defect-free & 27.387 & 11.005 & 9.6240 & 8.8359 & 9.8807 \\
\hline & & & Complete gap & 24.437 & 10.650 & 9.3505 & 8.6476 & 9.5668 \\
\hline & & & Complete & 37.407 & 12.844 & 11.0096 & 10.0458 & 12.615 \\
\hline & \multirow{4}{*}{10} & QI & - & 22.944 & 18.599 & 17.9207 & 17.4953 & 17.996 \\
\hline & & \multirow{3}{*}[\pm<58|39>]{$_{4 \mathrm{~s}}$} & Defect-free & 27.387 & 21.429 & 20.5281 & 19.9680 & 20.537 \\
\hline & & & Complete gap & 24.437 & 19.581 & 18.8261 & 18.3541 & 18.832 \\
\hline & & & Complete & 37.407 & 27.819 & 26.4589 & 25.6235 & 26.992 \\
\hline & \multirow{4}{*}{20} & QI & - & 22.944 & 21.678 & 21.4415 & 21.2866 & 21.465 \\
\hline & & \multirow{3}{*}[\pm<58|39>]{$_{4 \mathrm{~s}}$} & Defect-free & 27.387 & 25.657 & 25.3244 & 25.1071 & 25.312 \\
\hline & & & Complete gap & 24.437 & 23.051 & 22.7818 & 22.6059 & 22.772 \\
\hline & & & Complete & 37.407 & 34.468 & 33.9274 & 33.5763 & 34.133 \\
\hline & \multirow{4}{*}{200} & QI & - & 22.944 & 22.931 & 22.9289 & 22.9271 & 22.929 \\
\hline & & \multirow{3}{*}[\pm<58|39>]{$_{4 \mathrm{~s}}$} & Defect-free & 27.387 & 27.444 & 27.4410 & 27.4384 & 27.425 \\
\hline & & & Complete gap & 24.437 & 24.482 & 24.4798 & 24.4778 & 24.467 \\
\hline & & & Complete & 37.407 & 37.419 & 37.4134 & 37.4091 & 37.438 \\
\hline \multirow{4}{*}{$\bar{\omega}$} & & QI & - & 14.576 & 10.724 & 10.2568 & 9.9767 & 10.367 \\
\hline & 5 & {$[ \pm<58 \mid 39>]_{4 \mathrm{~s}}$} & Defect-free & 15.925 & 11.205 & 10.6708 & 10.3534 & 10.747 \\
\hline & & & Complete gap & 15.283 & 11.064 & 10.5641 & 10.2655 & 10.634 \\
\hline & & & Complete & 17.762 & 11.863 & 11.2461 & 10.8838 & 11.640 \\
\hline
\end{tabular}




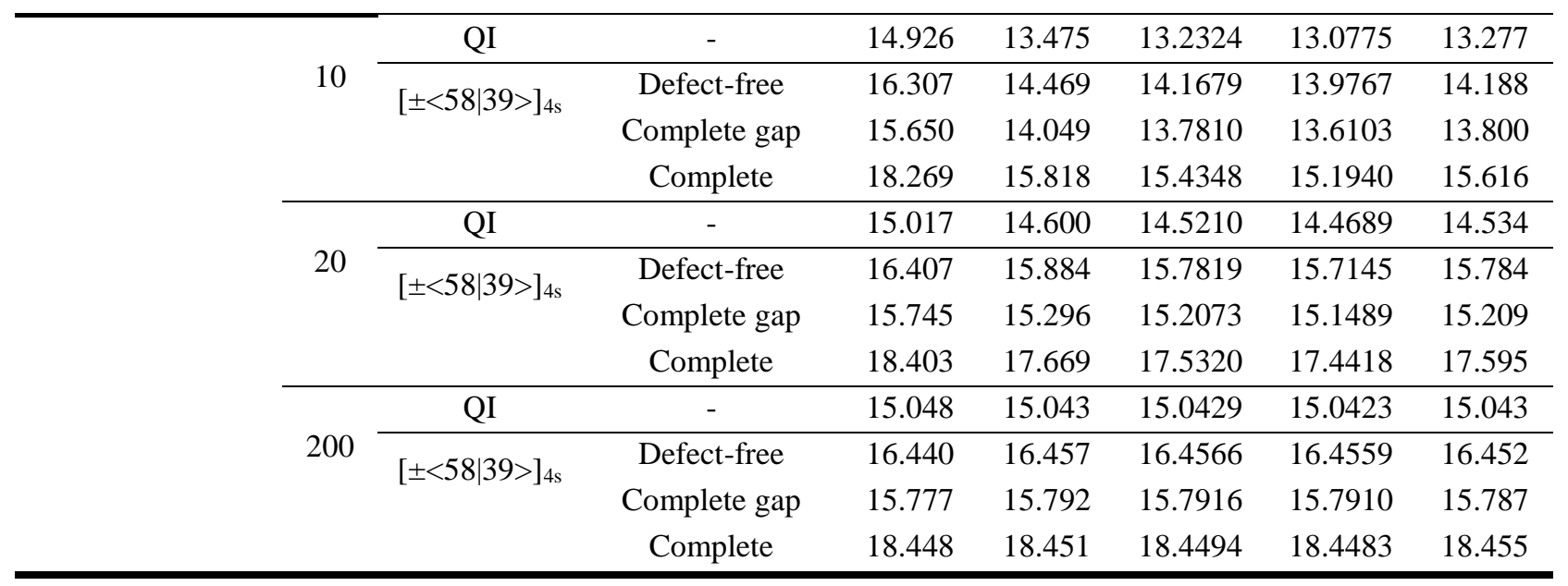




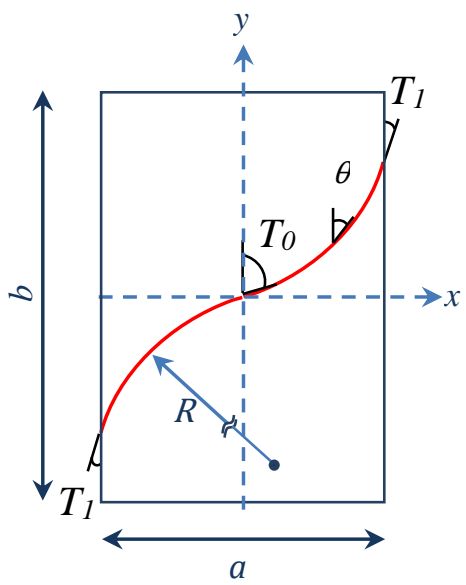

(a)

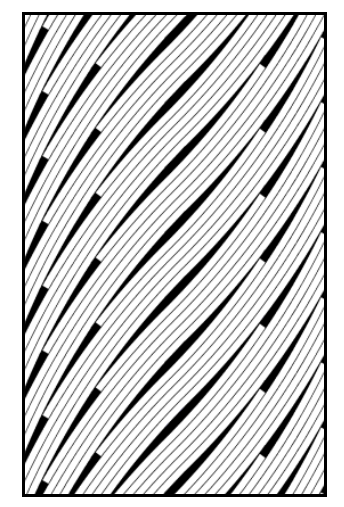

(b)

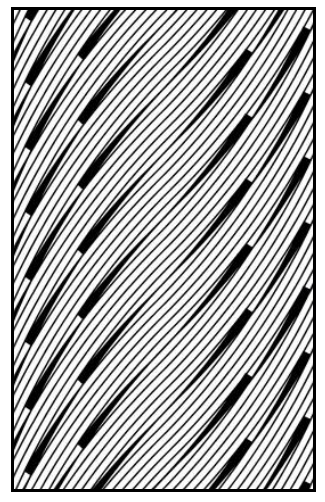

(c)

Fig. 1: (a) Fiber path definition; (b) gap (shaded area) distribution within the laminate; (c) overlap (shaded area) distribution within the laminate. 


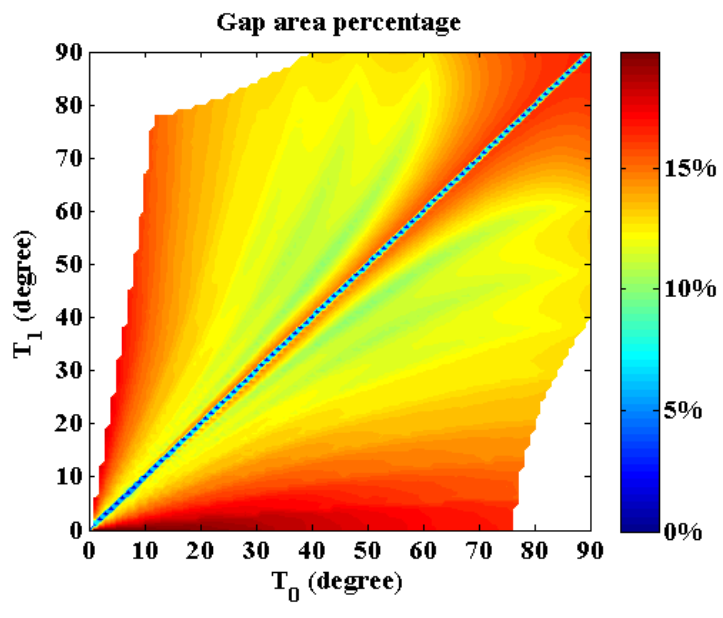

(a)

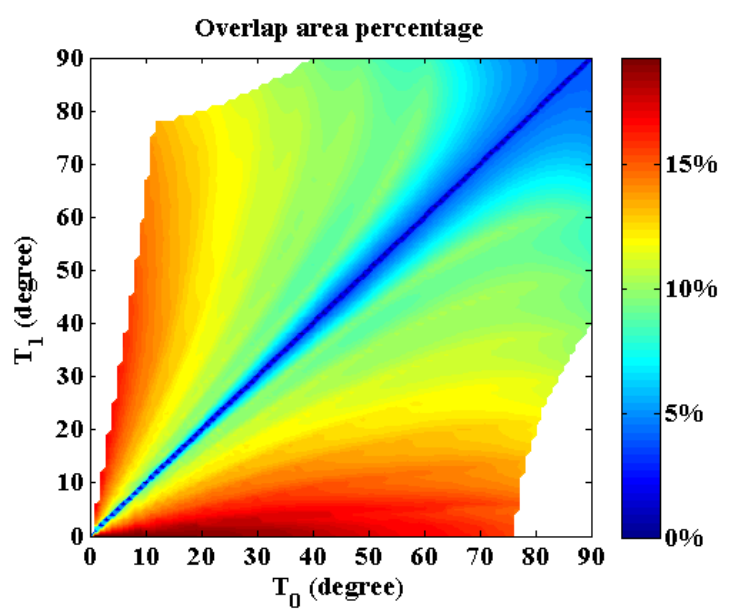

(b)

Fig. 2: (a) Complete gap area percentage and (b) complete overlap area percentage as a function of $T_{0}$ and $T_{1}$ for a variable stiffness plate. 


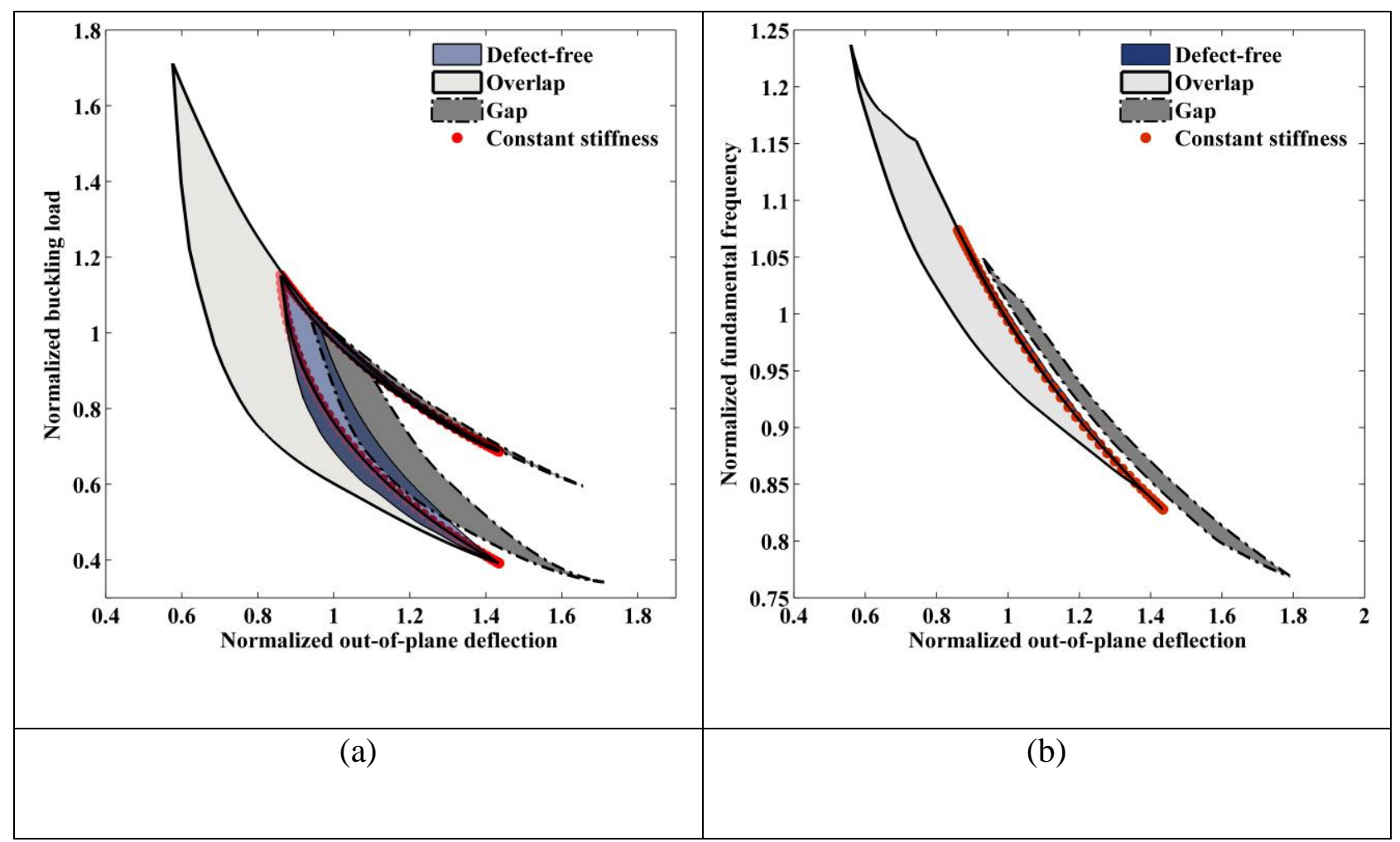

Fig. 3: Plate response domains for (a) buckling-deflection and (b) fundamental frequency-deflection in different manufacturing scenarios. 